INPLASY

PROTOCOL

To cite: Gao et al.

Effectiveness of montelukast for uremic pruritus in

hemodialysis patients: A

protocol for systematic review

and meta-analysis. Inplasy

protocol 2020100043. doi:

10.37766/inplasy2020.10.0043

Received: 13 October 2020

Published: 13 October 2020

Corresponding author:

Ya-yin Tan

tan574063928171@126.com

Author Affiliation:

Hemopurification Center,

Yijishan Hospital of Wannan

Medical College, Wuhu, China

Support: WSTP (2020rkx4-4).

Review Stage at time of this submission: The review has not yet started.

Conflicts of interest:

None.

\section{Effectiveness of montelukast for uremic pruritus in hemodialysis patients: A protocol for systematic review and meta-analysis}

Gao, CQ1; Zhou, JJ2; Tan, YY3; Tong, CJ4.

Review question / Objective: To evaluate effectiveness of montelukast for UP in hemodialysis patients.

Condition being studied: Montelukast for uremic pruritus in hemodialysis patients.

Information sources: The following electronic databases were searched: Pubmed, Embase, Web of Science, Cochrane Library, the China National Knowledge Infrastructure, Chinese Biomedical Literature Database, and China Science and Technology Journal Database. The range of publication time was from the inception of the database to December 2020. The similar search strategies will be used for other electronic databases.

INPLASY registration number: This protocol was registered with the International Platform of Registered Systematic Review and Meta-Analysis Protocols (INPLASY) on 13 October 2020 and was last updated on 13 October 2020 (registration number INPLASY2020100043).

\section{INTRODUCTION}

Review question / Objective: To evaluate effectiveness of montelukast for UP in hemodialysis patients.

Condition being studied: Montelukast for uremic pruritus in hemodialysis patients.

\section{METHODS}

Participant or population: Hemodialysis patients with UP will be included without restrictions of the nationality, age, gender, and race.

Intervention: In the treatment group, patients were given montelukast with no 
limitations of dosage and duration of intervention.

Comparator: RCTs that have control groups with conventional medication treatments or placebo will be included.

Study designs to be included: Randomized controlled trials (RCTs) of montelukast for UP in hemodialysis patients will be considered for inclusion without language limitation. The included trials were required to contain statistical methods and accurate data. Duplicate studies, animal experiments, reviews or case reports were excluded.

Eligibility criteria: RCTs of montelukast for UP in hemodialysis patients will be included.

Information sources: The following electronic databases were searched: Pubmed, Embase, Web of Science, Cochrane Library, the China National Knowledge Infrastructure, Chinese Biomedical Literature Database, and China Science and Technology Journal Database. The range of publication time was from the inception of the database to December 2020. The similar search strategies will be used for other electronic databases.

Main outcome(s): Pruritus severity as assessed using a Visual Analogue Scale (VAS), and the Detailed Pruritus Scale will be designated as the primary outcomes.

Additional outcome(s): Secondary outcome will included adverse events due to the medication.

Quality assessment / Risk of bias analysis: The Cochrane Collaboration tool will be used to assess the risk of bias of the selected studies. The following aspects were assessed independently by two reviews: random sequence generation, allocation concealment, blinding of participants and personnel, blinding of outcome assessment, incomplete outcome data, selective reporting, and other bias. Disagreements were analyzed by the third reviewer.
Strategy of data synthesis: Data synthesis will be performed by Review Manager 5.3. Continuous outcomes will be used for mean difference with $95 \%$ confidence interval. Risk ratio will be used for dichotomous outcomes with $95 \%$ confidence interval. Heterogeneity will be examined using the 12 test. If the 12 value > $50 \%$, the random effects model will be used. Otherwise, the fixed effects model will be utilized. If significant heterogeneity still exists after subgroup analysis, descriptive summary will be reported.

Subgroup analysis: If included studies have greater heterogeneity, subgroup analysis will be conducted to explore potential sources of heterogeneity. Subgroup analysis will be divided by different participant characteristics, disease course, controls, interventions and outcome measures.

Sensibility analysis: Sensitivity analysis will be applied to check the robustness and reliability of pooled results made in the review process. We will perform metaanalysis again after deleting low-quality studies and apply different statistical methods.

\section{Country(ies) involved: China.}

Keywords: montelukast, uremic pruritus, hemodialysis, protocol, systematic review.

Contributions of each author:

Author 1 - Chao-qing Gao.

Author 2 - Jia-jun Zhou.

Author 3 - Ya-yin Tan.

Author 4 - Chang-jun Tong. 